\title{
The Impact of Awareness Programs with Recruitment and Delay on the Spread of an Epidemic
}

\author{
Lixia Zuo, Maoxing Liu, and Jinqiang Wang \\ Department of Mathematics, North University of China, Taiyuan, Shanxi 030051, China \\ Correspondence should be addressed to Maoxing Liu; liumxsx@gmail.com
}

Received 2 April 2014; Revised 18 July 2014; Accepted 1 August 2014

Academic Editor: Yuxin Zhao

Copyright (c) 2015 Lixia Zuo et al. This is an open access article distributed under the Creative Commons Attribution License, which permits unrestricted use, distribution, and reproduction in any medium, provided the original work is properly cited.

\begin{abstract}
A compartment epidemic model with delay is given to discuss the impact of awareness programs on the spread and control of infectious diseases in a given region. It is assumed that there is a constant recruitment rate in the cumulative density of awareness programs, and further it is assumed that awareness programs can influence the susceptible to a limited extent. The system exhibits two equilibria: the disease-free equilibrium is stable if the basic reproduction number is less than unity for any delay and the unique endemic equilibrium exhibits Hopf-bifurcation under certain conditions. Numerical simulations prove the results of analysis and the significance of awareness programs in preventing and controlling the diseases, by investigating the relationship between the proportion of the infective and the dissemination rate and the implementation rate, respectively.
\end{abstract}

\section{Introduction}

Plenty of evidence shows that awareness programs, which can influence the susceptible to a limited extent due to some objective factors, play an important role in the spread and control of infectious diseases. For example, during the outbreak of SARS, H1N1 influenza pandemic, and HIV epidemic, public media had massive reports on the number of the infections and deaths per day, which had a great impact on the diseases control [1-4]. That is due to the fact that the spread of diseases is often accompanied by a rise in awareness of those in the social vicinity of infected individuals and a subsequent change in behavior, such as keeping social distancing, wearing protective masks, and vaccination $[5,6]$. Such reactions can manifest themselves in lower susceptibility as people try to prevent themselves from catching the disease, but also in lower infectivity because of self-imposed quarantine or better hygiene, shorter durations of infectiousness, or longer immunity [7]. And once the infective are cured, they will be aware of the disease [8].

Recently some scholars used mathematical models to discuss the impact of awareness programs on the diseases spreading and controlling in a given region [6, 8-11]. Joshi et al. [9] formulated a model to investigate the effect on the HIV epidemic in Uganda and compared their model with three types of the susceptible to a standard SIR model and then pointed out that the awareness programs in Uganda are successful in combating diseases. Li and Cui [1] analyzed a SIS epidemic model incorporating media coverage under constant and pulse vaccination; then they obtained the exact periodic infection-free solution which is globally asymptotically stable under some conditions. In order to better describe populations mixed condition, some authors studied infectious diseases models on different networks [1214]. In [12], the authors introduced a constant to represent the density level of media coverage from other regions. Funk et al. [14] formulated and analyzed a mathematical model in a host population; then they put forward that awareness programs can result in a lower size of the outbreak but do not affect the epidemic threshold, and if the behavioral response is treated as a local effect arising in the proximity of an outbreak, it can completely stop a disease from spreading, although only if the infection rate is below a threshold.

Some scholars consider some other factors in awareness program models. Liu and Wang et al. took into account the random perturbation $[15,16]$. In [16], the authors extended a deterministic SIRS epidemic model to a stochastic differential equation, and then they discussed the exponential $p$-stability and global stability of unique positive solution. Some scholars 
focused their attention at the contact rate, and most of them assumed that awareness programs will aid in modifying the contact rate between the susceptible and the infective [1729]. Liu and Cui [17] used the contact rate $\left(\beta_{1}-\beta_{2} I /(m+I)\right)$ and found that both of the two equilibria are asymptotically stable. Mirsa et al. [20] used the constant $k$ with the contact rate $M /(k+M)$ to limit the effect of awareness programs on the susceptible and sought out the conditions that Hopfbifurcation occurs. Tchuenche and Bauch [23] used an exponentially decreasing function $e^{-M(t)}$ to affect the force of infection; then numerical results showed the potential shortterm beneficial effect of awareness programs. Pang and Cui [26] used $\beta(I)=\mu_{1}-\mu_{2} f(I)$ to show the contact rate after awareness programs alerts and found that though it is not a determined fact to eradicate the infection of the diseases, the effective awareness programs can postpone the arrival of the infection peak. Elenbaas et al. [28] introduced a segmented function to describe the media impact $e^{-m I_{c}}$ when they formulate an epidemic model. A Filippov epidemic model was proposed to describe the real characteristics of media impact on the spread of infectious diseases by incorporating a piecewise continuous transmission rate $\beta e^{(-\alpha \epsilon I)} S I$ in [29]. Mathematical and bifurcation analyses with regard to the local and global stability of equilibria and local sliding bifurcations are performed.

But what we regard as unreasonable is that most of the articles assume that the cured infective become unaware of the disease. In fact people have a certain consciousness about the disease once they get sick. Therefore we propose a delayed mathematical model for predicting the future course of any epidemic by considering some of the infect join the aware susceptible after recovery. We hold the opinion that awareness programs can influence the susceptible to a limited extent for some objective factors and then consider the interaction between the susceptible and awareness programs as Holling type-II functional response. In addition we make a constant to represent the density level of media coverage from other regions with the disease because other regions can also effect the region that we consider. In fact the results about global stability of other delayed systems could be further utilized for other related problems [30-32].

The rest of this paper is organized as follows. In the next section, a mathematical model with delay has been proposed to capture the dynamics of the effect of awareness programs. It is assumed that diseases spread due to the contact between the susceptible and the infective only. Then we analyze the conditions of the stability of equilibria and the existence of Hopf-bifurcation in Section 3. Furthermore, in Section 4 we perform some numerical examples to validate the analysis in Section 3 and then introduce the importance of the dissemination rate, implementation rate, and the delay in disease control. In Section 5 we discuss the above contents.

\section{Mathematical Model and Equilibrium Analysis}

In the region under consideration the rate of immigration of the susceptible is $b$. It is assumed that the disease spreads due to the direct contact between the susceptible and the infective only and due to awareness programs the susceptible avoid being in contact with the infective and form a different class with a proportion $\lambda$, named the aware susceptible. So the total population is divided into three classes: the susceptible, the aware susceptible, and the infective, the proportions of which at time $t$ in the total population are $X(t), X_{m}(t)$, and $Y(t)$. It is assumed that the aware susceptible may lose awareness with passage of time and become susceptible with the proportion of $\lambda_{0}$ again. The infective can be cured with a proportion $\nu$ and a fraction $q$ of recovered people will become aware and join the aware susceptible class whereas the remaining fraction $p(p+q=1)$ will join the susceptible.

Consider that the cumulative density of awareness programs driven by media in that region at time $t$ is $M(t)$, which is related to the infective. We make the constant $\mu$ represent the executed rate of awareness programs. As the time passes, some campaigns lose their impact on people and lead to the diminution of the awareness programs, so we introduce $\mu_{0}$ to denote the rate of their depletion. Moreover $m_{0}$ represents the density level of media coverage on the disease from other regions. Using the fact that there is a limited extent of the awareness programs influence on the susceptible due to some objective factors, we introduce $k$ to limit the effect and consider the interaction between them as Holling type-II functional response. It is plausible that the policy makers need some time to gather the cases of the infective generally; then we introduce $\tau$ and consider that the cumulative density of awareness programs at time $t$ being executed will be in accordance with the infected cases reported at time $t-\tau(\tau>0)$. Keeping the above facts in mind, the dynamics of model is governed by the following system of nonlinear delay differential equations:

$$
\begin{aligned}
X^{\prime}(t)= & b-\beta X(t) Y(t)+\lambda_{0} X_{m}(t) \\
& -\lambda X(t) \frac{M(t)}{1+k M(t)}+p \nu Y(t)-d X(t), \\
X_{m}^{\prime}(t)= & \lambda X(t) \frac{M(t)}{1+k M(t)} \\
& -\lambda_{0} X_{m}(t)+q \nu Y(t)-d X_{m}(t), \\
Y^{\prime}(t)= & \beta X(t) Y(t)-\nu Y(t)-d Y(t), \\
M^{\prime}(t)= & m_{0}+\mu Y(t-\tau)-\mu_{0} M(t) .
\end{aligned}
$$

Here $X(0)=X_{0}>0, X_{m}(0)=X_{m 0} \geq 0$, and $Y(\theta)=Y_{0} \geq 0$ for $\theta \in[-\tau, 0]$ and $M(0)=M_{0} \geq 0$.

In the above model, the constants $\beta$ and $d(d=b)$, respectively, represent the contact rate of the unaware susceptible with the infective and the natural death rate. All the constants in the system are assumed to be positive. 
Using the fact that $X(t)+Y(t)+X_{m}(t)=1, p+q=1$ system (1) is reduced to the following system:

$$
\begin{aligned}
X_{m}^{\prime}(t)= & \lambda\left(1-X_{m}(t)-Y(t)\right) \frac{M(t)}{1+k M(t)} \\
& -\left(\lambda_{0}+d\right) X_{m}(t)+q \nu Y(t), \\
Y^{\prime}(t)= & \beta\left(1-X_{m}(t)-Y(t)\right) Y(t)-(\nu+d) Y(t), \\
M^{\prime}(t)= & m_{0}+\mu Y(t-\tau)-\mu_{0} M(t) .
\end{aligned}
$$

Now it is sufficient to study system (2) in detail rather than system (1).

For the analysis of system (2), we need the region of attraction which is given by the set $\Omega=\left\{\left(X_{m}, Y, M\right) \in \mathfrak{R}_{+}^{3}\right.$ : $\left.0 \leq X_{m}, Y \leq 1,0 \leq M \leq\left(m_{0}+\mu\right) / \mu_{0}\right\}$, and it attracts all solutions initiating in the interior of the positive orthant.

The system (2) has two equilibria.

(i) Disease-free equilibrium $E_{0}\left(\lambda m_{0} /\left(\lambda m_{0}+\left(\lambda_{0}+d\right)\left(\mu_{0}+\right.\right.\right.$ $\left.\left.\left.k m_{0}\right)\right), 0, m_{0} / \mu_{0}\right)$.

(ii) Endemic equilibrium $E_{*}\left(X_{m}^{*}, Y^{*}, M^{*}\right)$.

Define the basic reproduction number $R_{0}=\beta /(\nu+d)$. The existence of equilibrium $E_{0}$ is trivial; then we prove the existence of $E_{*}$ in detail. When $R_{0}>1$, in the equilibrium $E_{*}$ the values of $X_{m}^{*}, M^{*}$ are

$$
\begin{aligned}
& X_{m}^{*}=1-\frac{\nu+d}{\beta}-Y^{*}, \\
& M^{*}=\frac{m_{0}}{u_{0}}+\frac{\mu}{\mu_{0}} Y^{*},
\end{aligned}
$$

with $Y^{*}$ satisfying the equation

$$
A_{1} Y^{* 2}+A_{2} Y^{*}+A_{3}=0
$$

where

$$
\begin{aligned}
A_{1}= & \mu k \beta\left(\lambda_{0}+d+q \nu\right), \\
A_{2}= & \mu k\left(\lambda_{0}+d\right)(\nu+d-\beta)+\lambda \mu(\nu+d) \\
& +\beta\left(\lambda_{0}+d+q \nu\right)\left(\mu_{0}+k m_{0}\right), \\
A_{3}= & \lambda m_{0}(\nu+d)+\left(\lambda_{0}+d\right)(\nu+d-\beta)\left(\mu_{0}+k m_{0}\right) .
\end{aligned}
$$

Solving (4) we get

$$
Y^{*}=\frac{-A_{2} \pm \sqrt{A_{2}^{2}-4 A_{1} A_{3}}}{2 A_{1}} .
$$

We obtain $A_{1}>0$, and when $\beta \lambda<k\left(\lambda_{0}+d\right)(\beta-\nu-d)$,

$$
\begin{aligned}
A_{3}= & \lambda m_{0}(\nu+d)+\left(\lambda_{0}+d\right)(\nu+d-\beta)\left(\mu_{0}+k m_{0}\right) \\
= & \beta \lambda m_{0}\left(\frac{1}{R_{0}}-1\right)+\beta \lambda m_{0} \\
& +\beta\left(\lambda_{0}+d\right)\left(\mu_{0}+k m_{0}\right)\left(\frac{1}{R_{0}}-1\right) \\
= & \beta \lambda m_{0}\left(\frac{1}{R_{0}}-1\right)+\beta \mu_{0}\left(\lambda_{0}+d\right)\left(\frac{1}{R_{0}}-1\right) \\
& +\beta m_{0}\left[\lambda-k\left(\lambda_{0}+d\right)\left(1-\frac{1}{R_{0}}\right)\right]<0,
\end{aligned}
$$

then we get $Y^{*}=\left(-A_{2}+\sqrt{A_{2}^{2}-4 A_{1} A_{3}}\right) / 2 A_{1}$ for $Y^{*}>0$.

Remark 1. From the expression of $Y^{*}$, it is easy to note that $d Y^{*} / d \lambda<0$ and $d Y^{*} / d \mu<0$, which shows that the equilibrium proportion of the infective decreases as the rate of dissemination of awareness among the susceptible and the implementation rate of awareness programs increases.

\section{Stability Analysis}

In this section we present the local stability of $E_{0}, E_{*}$ and explore the conditions of Hopf-bifurcation by taking delay $\tau$ as a bifurcation parameter.

\subsection{Stability of Equilibria without Delay $(\tau=0)$}

Theorem 2. The disease-free equilibrium $E_{0}$ is locally asymptotically stable if $R_{0}<1$.

Proof. The Jacobian matrix corresponding to the system (2) when $\tau=0$ is given below:

$$
J=\left[\begin{array}{ccc}
-\frac{\lambda M}{1+k M}-\lambda_{0}-d & -\frac{\lambda M}{1+k M}+\nu q & \lambda\left(1-Y-X_{m}\right) \frac{1}{(1+k M)^{2}} \\
-\beta Y & \beta\left(1-X_{m}\right)-(\nu+d)-2 \beta Y & 0 \\
0 & \mu
\end{array}\right] .
$$


The characteristic equation at $E_{0}$ is of the form

$$
\begin{gathered}
\left(\eta+\mu_{0}\right)\left[\eta+\frac{\lambda m_{0}}{k m_{0}+\mu_{0}}+\lambda_{0}+d\right] \\
\times\left[\eta+\frac{\beta \lambda m_{0}}{\lambda m_{0}+\left(\lambda_{0}+d\right)\left(\mu_{0}+k m_{0}\right)}\right. \\
\left.+\beta\left(\frac{1}{R_{0}}-1\right)\right]=0,
\end{gathered}
$$

where $\eta$ is the eigenvalue. We get

$$
\begin{aligned}
& \eta_{1}=-\mu_{0}<0, \\
& \eta_{2}=-\left(\frac{\lambda m_{0}}{k m_{0}+\mu_{0}}+\lambda_{0}+d\right)<0, \\
& \eta_{3}=-\beta\left[\frac{\lambda m_{0}}{\lambda m_{0}+\left(\lambda_{0}+d\right)\left(\mu_{0}+k m_{0}\right)}+\left(\frac{1}{R_{0}}-1\right)\right],
\end{aligned}
$$

so $\eta_{3}<0$ when $R_{0}<1$. Thus $E_{0}$ is locally asymptotically stable if $R_{0}<1$.

The form of characteristic equation at $E_{*}$ is

$$
\eta^{3}+Q_{1} \eta^{2}+Q_{2} \eta+\left(Q_{3}+Q_{4}\right)=0
$$

where

$$
\begin{aligned}
& Q_{1}=B_{1}+B_{3}+\mu_{0}, \\
& Q_{2}=\mu_{0}\left(B_{1}+B_{3}\right)+B_{1} B_{3}-\beta Y^{*} B_{2}, \\
& Q_{3}=\mu_{0} B_{1} B_{3}-\beta \mu_{0} Y^{*} B_{2}, \\
& Q_{4}=-\beta \mu Y^{*} B_{4}, \\
& B_{1}=\lambda M^{*} \frac{1}{1+k M^{*}}+\lambda_{0}+d, \\
& B_{2}=\lambda M^{*} \frac{1}{1+k M^{*}}-\nu q, \\
& B_{3}=\beta\left(X_{m}^{*}-1\right)+2 \beta Y^{*}+(\nu+d), \\
& B_{4}=\lambda\left(X_{m}^{*}+Y^{*}-1\right) \frac{1}{\left(1+k M^{*}\right)^{2}} .
\end{aligned}
$$

Theorem 3. When the endemic equilibrium $E_{*}$ exists, it is locally asymptotically stable provided $m_{0}\left(\lambda_{0}+d+q \nu\right)>\mu \mu_{0}$.
Proof. For the characteristic equation

$$
\eta^{3}+Q_{1} \eta^{2}+Q_{2} \eta+\left(Q_{3}+Q_{4}\right)=0,
$$

it is easy to show

$$
\begin{aligned}
Q_{1}= & \lambda M^{*} \frac{1}{1+k M^{*}}+\lambda_{0}+d \\
& +\beta\left(X_{m}^{*}-1\right)+2 \beta Y^{*}+(\nu+d)+\mu_{0} \\
= & \lambda M^{*} \frac{1}{1+k M^{*}}+\lambda_{0}+d+\beta\left(X_{m}^{*}-1\right) \\
& +2 \beta Y^{*}+\beta\left(1-X_{m}^{*}-Y^{*}\right)+\mu_{0} \\
= & \lambda M^{*} \frac{1}{1+k M^{*}}+\lambda_{0}+d+\beta Y^{*}+\mu_{0}>0, \\
Q_{2}= & \mu_{0}\left(B_{1}+B_{3}\right)+\left(\lambda M^{*} \frac{1}{1+k M^{*}}+\lambda_{0}+d\right) \\
& \times\left[\beta\left(X_{m}^{*}-1\right)+2 \beta Y^{*}+(\nu+d)\right] \\
& -\beta Y^{*}\left(\lambda M^{*} \frac{1}{1+k M^{*}}-v q\right) \\
= & \mu_{0}\left(B_{1}+B_{3}\right)+\beta Y^{*}\left(\frac{\lambda M^{*}}{1+k M^{*}}+\lambda_{0}+d\right)
\end{aligned}
$$

$$
\begin{aligned}
& -\beta Y^{*}\left(\frac{\lambda M^{*}}{1+k M^{*}}-v q\right) \\
= & \mu_{0}\left(B_{1}+B_{3}\right)+\left(\lambda_{0}+v q+d\right) \beta Y^{*}>0,
\end{aligned}
$$

$$
Q_{3}+Q_{4}=\mu_{0} B_{1} B_{3}-\beta Y^{*}\left(\mu B_{4}+\mu_{0} B_{2}\right)
$$$$
=\mu_{0} \beta Y^{*}\left(\lambda M^{*} \frac{1}{1+k M^{*}}+\lambda_{0}+d\right)
$$$$
-\beta Y^{*}\left[\mu \lambda\left(X_{m}^{*}+Y^{*}-1\right) \frac{1}{\left(1+k M^{*}\right)^{2}}\right.
$$$$
\left.+\mu_{0}\left(\lambda M^{*} \frac{1}{1+k M^{*}}-v q\right)\right]
$$$$
=\beta Y^{*} \mu \lambda\left(1-X_{m}^{*}-Y^{*}\right) \frac{1}{\left(1+k M^{*}\right)^{2}}
$$$$
+\mu_{0} \beta Y^{*}\left(\lambda_{0}+d\right)+v q \mu_{0} \beta Y^{*}>0 .
$$

And

$$
\begin{aligned}
Q_{1} Q_{2} & -\left(Q_{3}+Q_{4}\right) \\
= & \left(B_{1}+B_{3}\right)\left(\mu_{0} B_{1}+\mu_{0} B_{3}+B_{1} B_{3}-\beta Y^{*} B_{2}\right) \\
& +\mu_{0}^{2}\left(B_{1}+B_{3}\right)-Q_{4}
\end{aligned}
$$




$$
\begin{aligned}
= & \mu_{0}\left(B_{1}+B_{3}\right)^{2}+\mu_{0}^{2}\left(B_{1}+B_{3}\right) \\
& +B_{3}\left[\left(B_{1}+B_{3}\right)\left(B_{1}-B_{2}\right)+\mu B_{4}\right],
\end{aligned}
$$

where

$$
\begin{aligned}
\left(B_{1}+\right. & \left.B_{3}\right)\left(B_{1}-B_{2}\right)+\mu B_{4} \\
= & \left(\frac{\lambda M^{*}}{1+k M^{*}}+\lambda_{0}+d+\beta Y^{*}\right)\left(\lambda_{0}+d+q \nu\right) \\
& -\mu \lambda\left(1-X_{m}^{*}-Y^{*}\right) \frac{1}{\left(1+k M^{*}\right)^{2}} \\
= & \frac{\lambda}{1+k M^{*}}\left[M^{*}\left(\lambda_{0}+d+q \nu\right)-\frac{\mu}{1+k M^{*}}\right] \\
& +\left(\lambda_{0}+d+\beta Y^{*}\right)\left(\lambda_{0}+d+q \nu\right)+\frac{\mu \lambda\left(X_{m}^{*}+Y^{*}\right)}{1+k M^{*}} \\
= & \frac{\lambda}{\left(1+k M^{*}\right)^{2}}\left[M^{*}\left(\lambda_{0}+d+q \nu\right)+k\left(M^{*}\right)^{2}\right. \\
& \quad+\left(\lambda_{0}+d+\beta Y^{*}\right)\left(\lambda_{0}+d+q \nu\right) \\
& +\mu \lambda\left(X_{m}^{*}+Y^{*}\right) \frac{1}{1+k M^{*}}
\end{aligned}
$$

$$
\begin{aligned}
& u(t)=\left[\begin{array}{lll}
x(t) & y(t) & z(t)
\end{array}\right]^{T}, \\
& M_{1}=\left[\begin{array}{ccc}
-\frac{\lambda M^{*}}{1+k M^{*}}-\lambda_{0}-d & -\frac{\lambda M^{*}}{1+k M^{*}}+\nu q & \lambda\left(1-Y^{*}-X_{m}^{*}\right) \frac{1}{\left(1+k M^{*}\right)^{2}} \\
-\beta Y^{*} & \beta\left(1-X_{m}^{*}\right)-(\nu+d)-2 \beta Y^{*} & 0 \\
0 & 0 & -\mu_{0}
\end{array}\right] \text {, } \\
& M_{2}=\left[\begin{array}{lll}
0 & 0 & 0 \\
0 & 0 & 0 \\
0 & \mu & 0
\end{array}\right] \text {. }
\end{aligned}
$$

$$
\begin{aligned}
& +\left(\lambda_{0}+d+\beta Y^{*}\right)\left(\lambda_{0}+d+q \nu\right) \\
& +\mu \lambda\left(X_{m}^{*}+Y^{*}\right) \frac{1}{1+k M^{*}}>0,
\end{aligned}
$$

so $Q_{1} Q_{2}-\left(Q_{3}+Q_{4}\right)>0$. According to Hurwitz criterion, we can claim that all the eigenvalues will be either negative or having negative real part. Thus, the equilibrium $E_{*}$ is locally asymptotically stable.

\subsection{Stability of Equilibria with Delay $(\tau>0)$}

Theorem 4. The disease-free equilibrium $E_{0}$ is locally asymptotically stable if $R_{0}<1$.

Similar to the proof of Theorem 2, this proof is omitted.

We linearize system (2) about $E_{*}$ to study the stability of the endemic equilibrium and get

$$
\frac{d u}{d t}=M_{1} u(t)+M_{2} u(t-\tau)
$$

where
In the above, $x, y$, and $z$ are small perturbations around $E_{*}$. is

Then the form of the characteristic equation of the system

$$
\eta^{3}+Q_{1} \eta^{2}+Q_{2} \eta+Q_{3}=Q_{4} e^{-\eta \tau}
$$

where $\eta$ is the eigenvalue.

To show the Hopf-bifurcation, we need to show that (19) has a pair of purely imaginary roots. For this, substituting $\eta=i \omega(\omega>0)$ into (19) and separating real and imaginary parts, we get the following transcendental equations:

$$
\begin{aligned}
& Q_{2} \omega-\omega^{3}=Q_{4} \sin (\omega \tau), \\
& Q_{1} \omega^{2}-Q_{3}=Q_{4} \cos (\omega \tau) .
\end{aligned}
$$

Squaring and adding the above equations and substituting $\psi=\omega^{2}$, we get

$$
h(\psi)=\psi^{3}+P_{1} \psi^{2}+P_{2} \psi+P_{3}=0,
$$

where $P_{1}=Q_{1}^{2}-2 Q_{2}, P_{2}=Q_{2}^{2}-2 Q_{1} Q_{3}$, and $P_{3}=Q_{3}^{2}-Q_{4}^{2}$. 
If the coefficients in $h(\psi)$ satisfy the conditions of RouthHurwitz criterion, then $E_{*}$ is locally asymptotically stable for all delays $\tau>0$, provided it is stable in absence of delay. In the following we consider that the values of $P_{i}(i=1,2,3,4)$ do not satisfy the Routh-Hurwitz criterion.

Lemma 5. If $B_{2}>0, \lambda_{0}+d+q \nu<\mu \lambda(\nu+d)\left(1 / \beta\left(1+k M^{*}\right)^{2}\right)$, (21) will have at least one positive root $\psi_{0}$.

Proof. For $h(\psi)=\psi^{3}+P_{1} \psi^{2}+P_{2} \psi+P_{3}$, it is obvious that

$$
\begin{aligned}
& P_{1}=\left(B_{1}+B_{3}+\mu_{0}\right)^{2} \\
& -2\left[\left(B_{1}+B_{3}\right) \mu_{0}+B_{1} B_{3}-\beta B_{2} Y^{*}\right] \\
& =B_{1}^{2}+B_{3}^{2}+\mu_{0}^{2}+2 \beta B_{2} Y^{*}>0, \\
& P_{2}=\left[\left(B_{1}+B_{3}\right) \mu_{0}+B_{1} B_{3}-\beta B_{2} Y^{*}\right]^{2} \\
& -2\left(B_{1}+B_{3}+\mu_{0}\right)\left(B_{1} B_{3} \mu_{0}-\mu_{0} \beta B_{2} Y^{*}\right) \\
& =B_{1}^{2} \mu_{0}^{2}+B_{3}^{2} \mu_{0}^{2}+\left(B_{1} B_{3}\right)^{2} \\
& +\left(B_{2} B_{3}\right)^{2}-2 B_{1} B_{2} B_{3}^{2}+2 B_{2} B_{3} \mu_{0}^{2} \\
& =\left(B_{1}^{2}+B_{3}^{2}\right) \mu_{0}^{2}+\left(B_{1} B_{3}-B_{2} B_{3}\right)^{2} \\
& +2 \mu_{0}^{2} B_{2} B_{3}>0 \\
& P_{3}=\left(Q_{3}+Q_{4}\right)\left(Q_{3}-Q_{4}\right) \\
& =B_{3}\left(\mu_{0} B_{1}-\mu_{0} B_{2}+\mu B_{4}\right)\left(Q_{3}+Q_{4}\right) \\
& =B_{3}\left(Q_{3}+Q_{4}\right) \\
& \times\left[\mu_{0}\left(\lambda_{0}+d+q v\right)\right. \\
& \left.-\mu \lambda\left(1-X_{m}^{*}-Y^{*}\right) \frac{1}{\left(1+k M^{*}\right)^{2}}\right] \\
& <B_{3}\left(Q_{3}+Q_{4}\right)\left[\left(\lambda_{0}+d+q v\right)\right. \\
& \left.-\mu \lambda(\nu+d) \frac{1}{\beta\left(1+k M^{*}\right)^{2}}\right]<0 .
\end{aligned}
$$

Then we have $h(0)<0$ and $h(+\infty) \rightarrow+\infty$; thus (21) has at least one positive root $\psi_{0}$.

Denote $\omega_{0}=\sqrt{\psi_{0}}$; then (21) has a pair of purely imaginary roots $\left( \pm i \omega_{0}\right)$.

Now we turn to the bifurcation analysis. We use the delay $\tau$ as the bifurcation parameter. We view the solutions of (21) as a function of the bifurcation parameter $\tau$, and let $\eta(\tau)=$ $\gamma(\tau)+i \omega(\tau)$ be the eigenvalue of (21) such that, for the initial value of the bifurcation parameter $\tau_{0}$, we have $\gamma\left(\tau_{0}\right)=0$ and $\omega\left(\tau_{0}\right)=\omega_{0}\left(\omega_{0}>0\right)$. To establish the Hopf bifurcation at $\tau=\tau_{0}$, we need to show that $d \operatorname{Re} \eta(\tau) /\left.d \tau\right|_{\tau=\tau_{0}}>0$.
Lemma 6. One has the following transversality condition:

$$
\left.\frac{d \operatorname{Re} \eta(\tau)}{d \tau}\right|_{\tau=\tau_{0}}>0
$$

Proof. Differentiating with respect to $\tau$ from (21), we get

$$
\left(\frac{d \eta}{d \tau}\right)^{-1}=\frac{d \tau}{d \eta}=\frac{3 \eta^{2}+2 Q_{1} \eta+Q_{2}-Q_{4} \tau e^{-\eta \tau}}{Q_{4} \eta e^{-\eta \tau}} .
$$

So

$$
\begin{aligned}
& \operatorname{Sign}\left\{\operatorname{Re} \frac{d(\eta)}{d(\tau)}\right\}_{\tau=\tau_{0}} \\
& =\operatorname{Sign}\left\{\operatorname{Re} \frac{d(\tau)}{d(\eta)}\right\}_{\eta=i \omega_{0}} \\
& =\operatorname{Sign}\left\{\operatorname{Re}\left[\frac{3 \eta^{2}+2 Q_{1} \eta+Q_{2}-Q_{4} \tau e^{-\eta \tau}}{Q_{4} \eta e^{-\eta \tau}}\right]_{\eta=i \omega_{0}}\right\} \\
& =\operatorname{Sign}\left\{\operatorname{Re}\left[\frac{3 \eta^{2}+2 Q_{1} \eta+Q_{2}}{Q_{4} \eta e^{-\eta \tau}}\right]_{\eta=i \omega_{0}}\right\} \\
& =\operatorname{Sign}\left\{\operatorname{Re}\left[-\frac{3 \eta^{2}+2 Q_{1} \eta+Q_{2}}{\eta^{4}+Q_{1} \eta^{3}+Q_{2} \eta^{2}+Q_{3} \eta}\right]_{\eta=i \omega_{0}}\right\} \\
& =\operatorname{Sign}\left\{\operatorname{Re}\left[-\frac{\left(Q_{2}-3 \omega_{0}^{2}\right)+2 Q_{1} \omega_{0} i}{\left(\omega_{0}^{4}-Q_{2} \omega_{0}^{2}\right)+\left(Q_{3} \omega_{0}-Q_{1} \omega_{0}^{3}\right) i}\right]\right\} \\
& =\operatorname{Sign}\left\{\frac{\omega_{0}^{2}\left[3 \omega_{0}^{4}+\left(2 Q_{1}^{2}-4 Q_{2}\right) \omega_{0}^{2}+\left(Q_{2}^{2}-2 Q_{1} Q_{3}\right)\right]}{Q_{4}^{2} \omega_{0}^{4}}\right\} \\
& =\operatorname{Sign}\left\{\frac{3 \omega_{0}^{4}+2 P_{1} \omega_{0}^{2}+P_{2}}{Q_{4}^{2}}\right\}>0 .
\end{aligned}
$$

This proves the lemma. Now we have the following theorem.

Theorem 7. The endemic equilibrium $E_{*}$ of the system is locally asymptotically stable when $\tau<\tau_{0}$ and becomes unstable for $\tau>\tau_{0}$ provided

$$
\frac{\mu \mu_{0}}{m_{0}}<\lambda_{0}+d+q \nu<\min \left\{\frac{\lambda M^{*}}{1+k M^{*}}, \frac{\mu \lambda(\nu+d)}{\beta\left(1+k M^{*}\right)^{2}}\right\} .
$$

When $\tau=\tau_{0}$, a Hopf-bifurcation occurs, leading a family of periodic solutions bifurcating from $E_{*}$ as $\tau$ passes through the critical value $\tau_{0}$, where

$$
\tau_{0}=\frac{1}{\omega_{0}} \arccos \frac{Q_{1} \omega_{0}^{2}-Q_{3}}{Q_{4}} .
$$



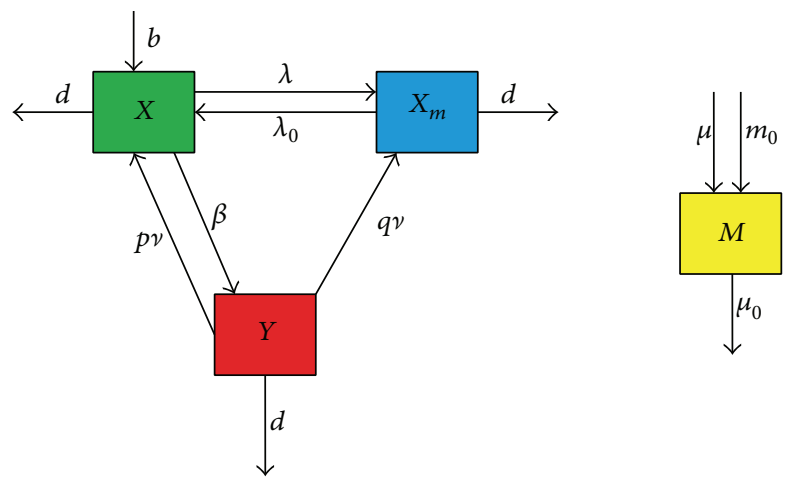

FIGURE 1: Schematic model flow diagram.

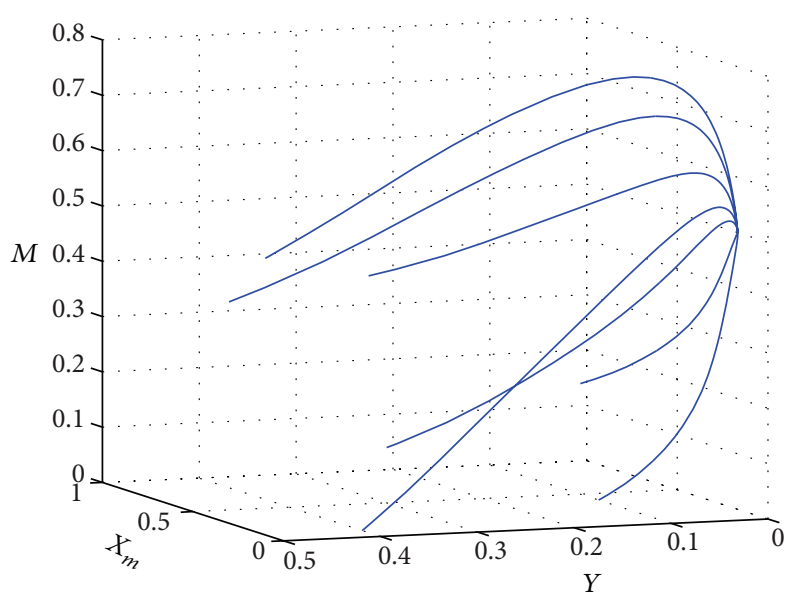

FIGURE 2: The stability of the disease-free equilibrium $E_{0}$ with $\tau=10$.

\section{Numerical Simulations and Results}

To check the feasibility of our analysis in Section 3 about $E_{0}$, we present some numerical computations in this section using MatLab by choosing the following set of parameter values: $d=0.002, \beta=0.4, \mu_{0}=0.08, \mu=0.2, \nu=0.46$, $q=0.6, \lambda=0.2, \lambda_{0}=0.4, m_{0}=0.04, k=0.5$, and $\tau=10$ (see Figure 1), and then we get Figure 2 with different initial values, from which we can know that $E_{0}$ is stable and all the trajectories approach it. The numerical simulations support the analysis given.

Then we choose the following set of parameter values which satisfy the condition in Theorem 7: $d=0.00004, \beta=$ $0.3, \mu_{0}=0.14, \mu=0.35, v=0.2, q=0.15, \lambda=0.019$, $\lambda_{0}=0.001, m_{0}=0.000005$, and $k=0.0003$. The numerical value of $\tau_{0}$ computed is found to be 30.12 . When $\tau>0$, we give different $\tau$ as follows: $\tau=20, \tau=25, \tau=35$, and $\tau=40$ (Figures 3 and 4). As shown in Figures 3 and 4 the variables approach their equilibria when $\tau$ is less than $\tau_{0}$, whereas, as $\tau$ exceeds its critical value $\tau_{0}$, all variables start showing oscillatory behavior. This indicates that, in the latter case, sometimes the infective will be high and sometimes will be low and it may be difficult to make the prediction regarding the size of epidemic. It is clear that $\tau$ plays a key role in the prevention and control of diseases.

In the following we let $\tau=10$ and make $\lambda, \mu$ change from 0.05 to 0.8 and research the influence of the dissemination rate $\lambda$ and the implementation rate $\mu$ on the infective $Y(t)$ separately. The variation of $Y(t)$ with respect to time $t$ for different values of $\lambda$ and $\mu$ is shown in Figure 5, and we discover all of the proportions of $Y^{*}$ reductions as $\lambda$ and $\mu$ increase, which proves the conclusions of the remark, but $\lambda$ has greater influence on $Y(t)$ than $\mu$. In addition the reason why $Y(t)$ has a similar trend in Figure 5 is that $\lambda$ and $\mu$ have a similar trend with $M(t)$. There really is an effort here to make clear that $\lambda$ and $\mu$ play a key role in the prevention and control of diseases.

A comparison between the oscillations in $Y$ and $M$ is presented in Figure 6. From this figure, we obtain that, as the infective increase, the awareness programs also start growing with some time lag due to time delay in the execution of awareness programs. As soon as the awareness programs reach a potentially high value, the infective start decreasing due to the execution of awareness programs in the infective. Awareness programs after certain time also start decreasing, which eventually results in the increase of the infective. Both 

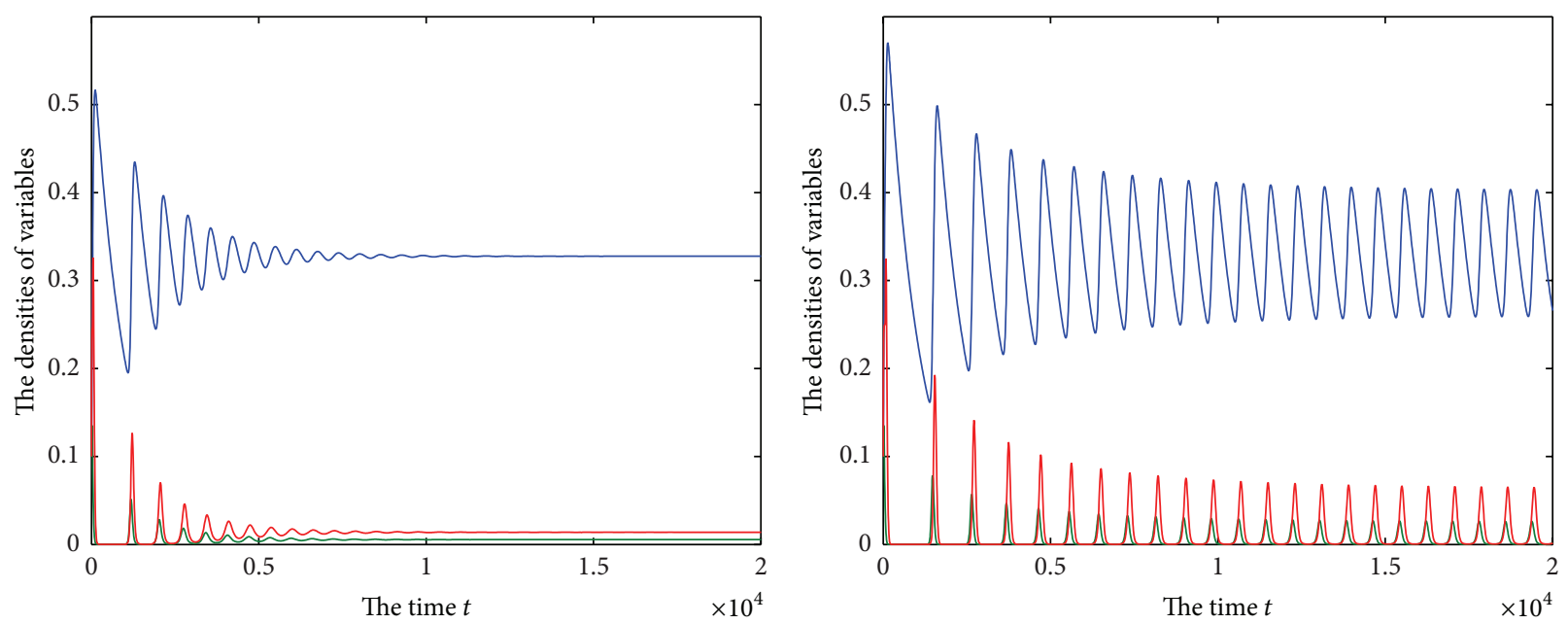
$-X_{m}$
$-X_{m}$
$Y$
$-M$

FIgURE 3: The stability of the endemic equilibria $E_{*}$ with $\tau=20$ and $\tau=40$, respectively.
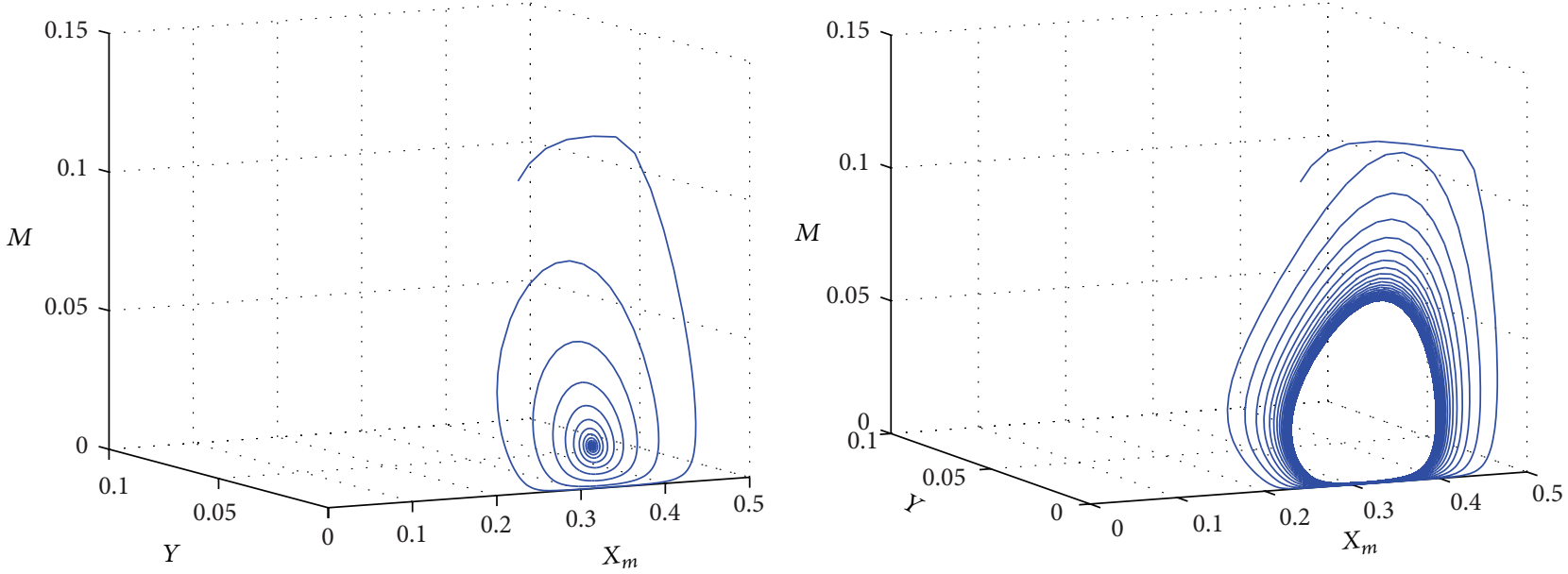

FIgURE 4: The stability of the endemic equilibria $E_{*}$ with $\tau=25$ and $\tau=35$, respectively.
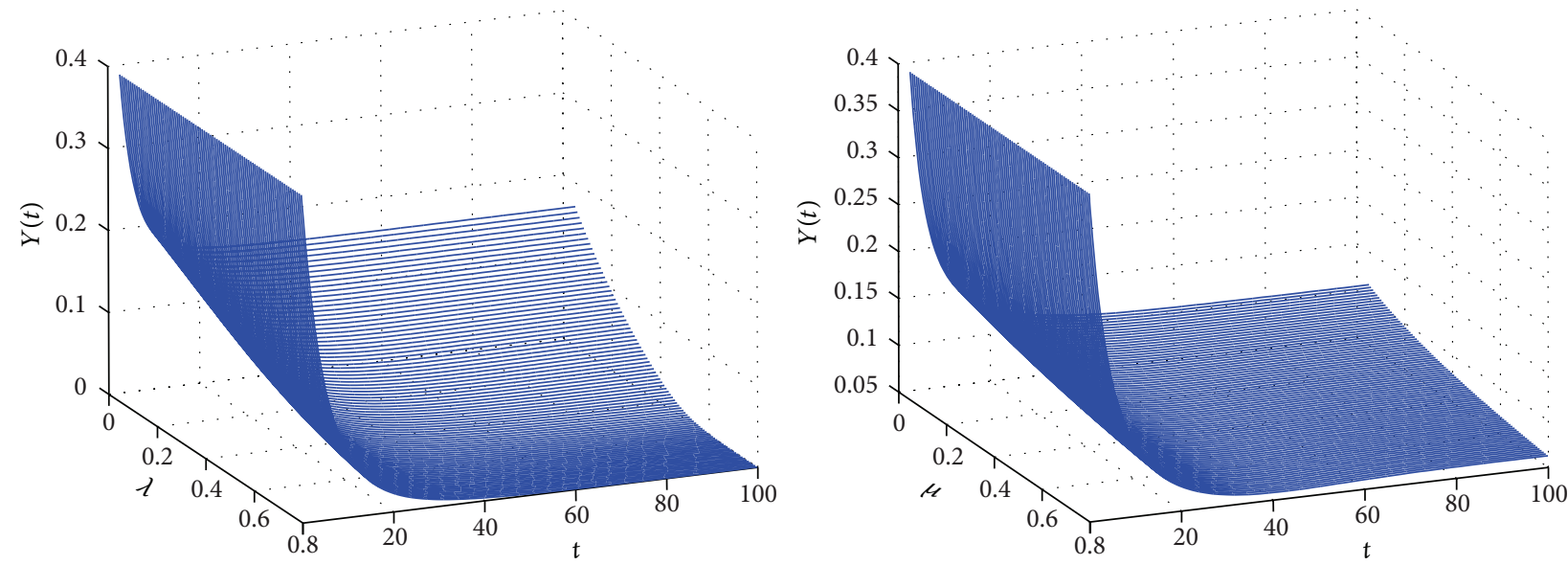

FIGURE 5: When $\tau=10$, the stability of $Y^{*}$ with variational $\lambda$ and $\mu$, respectively. 

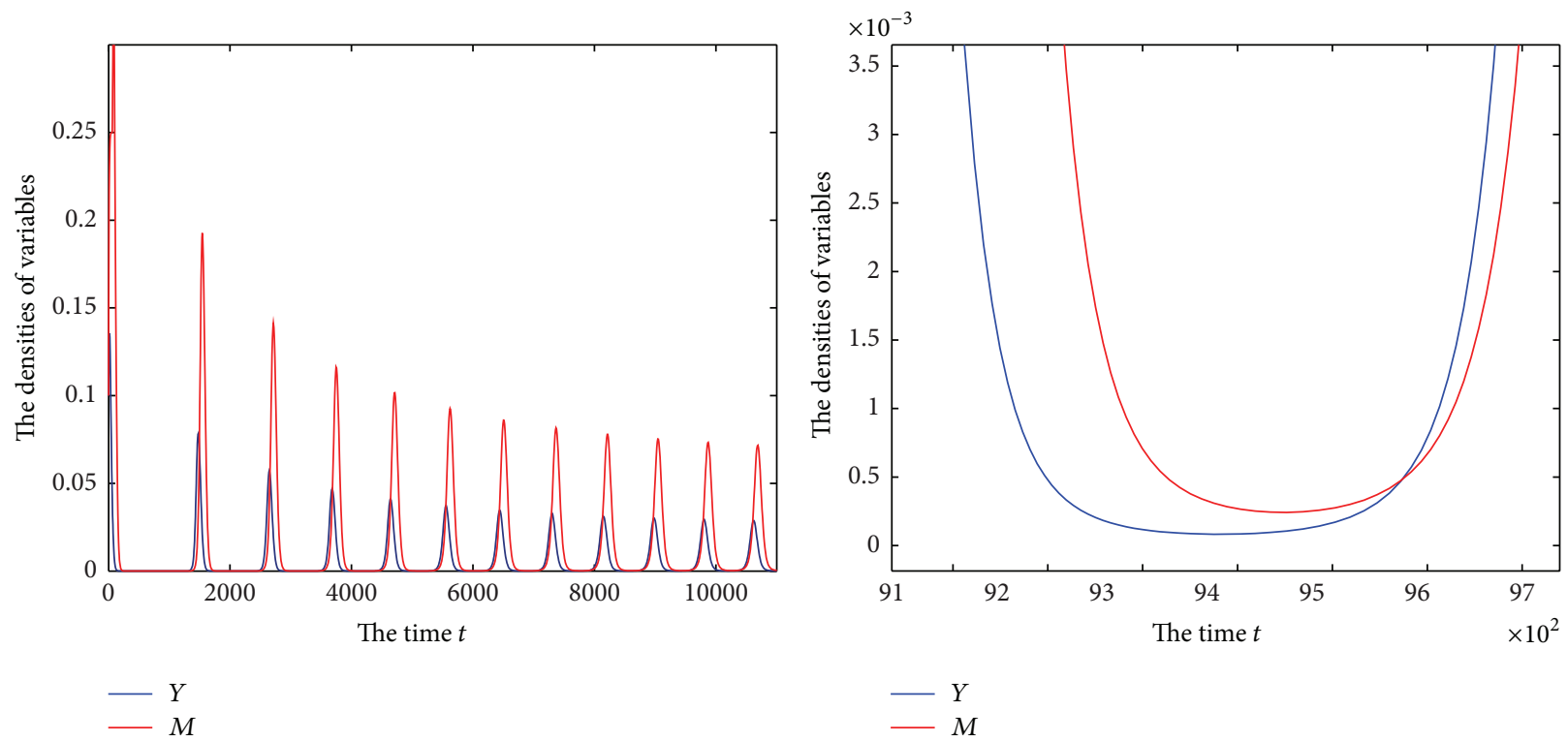

FIGURE 6: Comparison between variations of $Y$ and $M$ with time.

of the figures show the interplay between $Y$ and $M$, which proves the existence of delay.

\section{Discussion}

In this paper, a nonlinear mathematical model with delay and awareness programs driven by media has been proposed and analyzed. It is assumed that pathogens are transmitted via direct contact between the susceptible and the infective. The model exhibits two equilibria, and the disease-free equilibrium has been shown to be stable for basic reproduction number $R_{0}<1$ when $\tau \geq 0$. For $R_{0}>1$, it leads to the existence and stability of an endemic equilibrium under some conditions in absence of time delay. But for $\tau>0$ this equilibrium is locally asymptotically stable when the delay is suitably small; that is, $\tau<\tau_{0}$, while a loss of stability by a Hopf-bifurcation can occur as delay increases. Numerical simulations prove the stability of equilibria and show that the value of delay sustainable for the disease is 30.12 . The numerical results suggest that if we want to reduce the proportion of the infective, we can increase the dissemination rate $\lambda$ and implementation rate $\mu$. At last we obtain the existence of $\tau$ from Figure 6 . In short media is a key tool for influencing people behavior towards the disease to devise proper policies for controlling the epidemic.

\section{Conflict of Interests}

The authors declare that there is no conflict of interests regarding the publishing of this paper.

\section{Acknowledgments}

This paper is supported by a Project of the National Sciences Foundation of China (10901145, 11301491, and 11331009) and the National Sciences Foundation of Shanxi Province (2012011002-1).

\section{References}

[1] Y. Li and J. Cui, "The effect of constant and pulse vaccination on SIS epidemic models incorporating media coverage," Communications in Nonlinear Science and Numerical Simulation, vol. 14, no. 5, pp. 2353-2365, 2009.

[2] Z. Mukandavire, W. Garira, and J. M. Tchuenche, "Modelling effects of public health educational campaigns on HIV/AIDS transmission dynamics," Applied Mathematical Modelling: Simulation and Computation for Engineering and Environmental Systems, vol. 33, no. 4, pp. 2084-2095, 2009.

[3] M. Brodie, E. Hamel, L. A. Brady, J. Kates, and D. E. Altman, "AIDS at 21: Media Coverage of the HIV Epidemic 1981-2002," Princeton Survey Research Associates International, 2004.

[4] J. T. F. Lau, M. Lau, J. H. Kim, and H. Y. Tsui, "Impacts of media coverage on the community stress level in Hong Kong after the tsunami on 26 December 2004," Journal of Epidemiology \& Community Health, vol. 60, no. 8, pp. 675-682, 2006.

[5] J. Cui, Y. Sun, and H. Zhu, "The impact of media on the control of infectious diseases," Journal of Dynamics and Differential Equations, vol. 20, no. 1, pp. 31-53, 2008.

[6] J. Cui, X. Tao, and H. Zhu, "An SIS infection model incorporating media coverage," The Rocky Mountain Journal of Mathematics, vol. 38, no. 5, pp. 1323-1334, 2008.

[7] J. M. Tchuenche, N. Dube, C. P. Bhunu, R. J. Smith, and C. T. Bauch, "The impact of media coverage on the transmission dynamics of human influenza," BMC Public Health, vol. 11, supplement 2, article S5, 2011.

[8] S. Samanta, S. Rana, A. Sharma, A. K. Misra, and J. Chattopadhyay, "Effect of awareness programs by media on the epidemic outbreaks: a mathematical model," Applied Mathematics and Computation, vol. 219, no. 12, pp. 6965-6977, 2013.

[9] H. Joshi, S. Lenhart, and K. Albright, "Modeling the effect of information campaigns on the HIV epidemic in Uganda," 
Mathematical Biosciences and Engineering, vol. 5, no. 4, pp. 757770, 2008.

[10] F. Nyabadza, C. Chiyaka, Z. Mukandavire, and S. D. HoveMusekwa, "Analysis of an HIV/AIDS model with public-health information campaigns and individual withdrawal," Journal of Biological Systems, vol. 18, no. 2, pp. 357-375, 2010.

[11] A. K. Misra, A. Sharma, and J. B. Shukla, "Modeling and analysis of effects of awareness programs by media on the spread of infectious diseases," Mathematical and Computer Modelling, vol. 53, no. 5-6, pp. 1221-1228, 2011.

[12] Y. Wang, J. Cao, Z. Jin, H. Zhang, and G. Sun, "Impact of media coverage on epidemic spreading in complex networks," Physica A: Statistical Mechanics and its Applications, vol. 392, no. 23, pp. 5824-5835, 2013.

[13] X. Yuan, Y. Xue, and M. Liu, "Analysis of an epidemic model with awareness programs by media on complex networks," Chaos, Solitons and Fractals, vol. 48, no. 1, pp. 1-11, 2013.

[14] S. Funk, E. Gilad, C. Watkins, and V. A. A. Jansen, "The spread of awareness and its impact on epidemic outbreaks," Proceedings of the National Academy of Sciences of the United States of America, vol. 106, no. 16, pp. 6872-6877, 2009.

[15] W. B. Liu, "A SIRS epidemic model incorporating media coverage with random perturbation," Abstract and Applied Analysis, vol. 2013, Article ID 792308, 9 pages, 2013.

[16] L. Y. Wang, H. L. Huang, A. C. Xu, and W. M. Wang, "Stochastic extinction in an SIRS epidemic model incorporating media coverage," Abstract and Applied Analysis, vol. 2013, Article ID 891765, 8 pages, 2013.

[17] Y. Liu and J. Cui, "The impact of media coverage on the dynamics of infectious disease," International Journal of Biomathematics, vol. 1, no. 1, pp. 65-74, 2008.

[18] A. L. Wang and Y. N. Xiao, "A filippov system describing media effects on the spread of infectious diseases," Nonlinear Analysis: Hybrid Systems, vol. 11, pp. 84-97, 2014.

[19] S. Collinson and J. M. Heffernan, "Modelling the effects of media during an influenza epidemic," BMC Public Health, vol. 14, article 376, 2014.

[20] A. K. Misra, A. Sharma, and V. Singh, "Effect of awareness programs in controlling the prevalence of an epidemic with time delay," Journal of Biological Systems, vol. 19, no. 2, pp. 389402, 2011.

[21] Y. Wang, J. Cao, Z. Jin, H. Zhang, and G. Q. Sun, "Impact of media coverage on epidemic spreading in complex networks," Physica A: Statistical Mechanics and Its Applications, vol. 392, no. 23, pp. 5824-5835, 2013.

[22] Y. Y. Liu and Y. N. Xiao, "An epidemic model with saturated media/psychological impact," Applied Mathematics and Mechanics, vol. 34, no. 4, pp. 399-407, 2013.

[23] J. M. Tchuenche and C. T. Bauch, "Dynamics of an infectious disease where media coverage influences transmission," ISRN Biomathematics, vol. 2012, Article ID 581274, 10 pages, 2012.

[24] V. Capasso, Mathematical Structures of Epidemic Systems, vol. 97 of Lecture Notes in Biomathematics, Springer, Berlin, Germany, 1993.

[25] H. W. Hethcote and S. A. Levin, "Periodicity in epidemiological models," in Applied Mathematical Ecology (Trieste, 1986), vol. 18 of Biomathematics, pp. 193-211, Springer, Berlin, Germany, 1989.

[26] J. H. Pang and J. A. Cui, "An SIRS epidemiological model with nonlinear incidence rate incorporating media coverage," Information and Computing Science, vol. 3, pp. 116-119, 2009.
[27] M. Liu, G. Röst, and G. Vas, "SIS model on homogeneous networks with threshold type delayed contact reduction," Computers \& Mathematics with Applications, vol. 66, no. 9, pp. 15341546, 2013.

[28] M. Elenbaas, H. G. Boomgaarden, A. R. T. Schuck, and C. H. de Vreese, "The impact of media coverage and motivation on performance-relevant information," Political Communication, vol. 30, no. 1, pp. 1-16, 2013.

[29] W. M. Liu, H. W. Hethcote, and S. A. Levin, "Dynamical behavior of epidemiological models with nonlinear incidence rates," Journal of Mathematical Biology, vol. 25, no. 4, pp. 359380, 1987.

[30] L. Wu, X. Su, and P. Shi, "Sliding mode control with bounded $L_{2}$ gain performance of Markovian jump singular time-delay systems," Automatica, vol. 48, no. 8, pp. 1929-1933, 2012.

[31] F. Li, P. Shi, L. Wu, and X. Zhang, "Fuzzy-model-based Dstability and non-fragile control for discrete-time descriptor systems with multiple delays," IEEE Transactions on Fuzzy Systems, vol. 22, no. 4, pp. 1019-1025, 2014.

[32] F. Li, L. Wu, and P. Shi, "Stochastic stability of semi-Markovian jump systems with mode-dependent delays," International Journal of Robust and Nonlinear Control, 2013. 


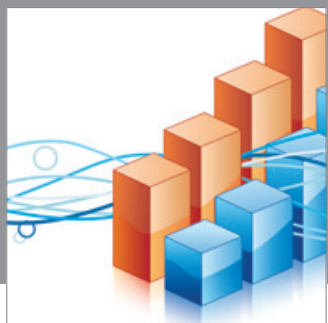

Advances in

Operations Research

mansans

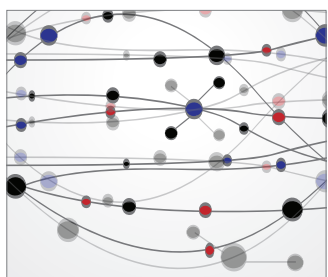

The Scientific World Journal
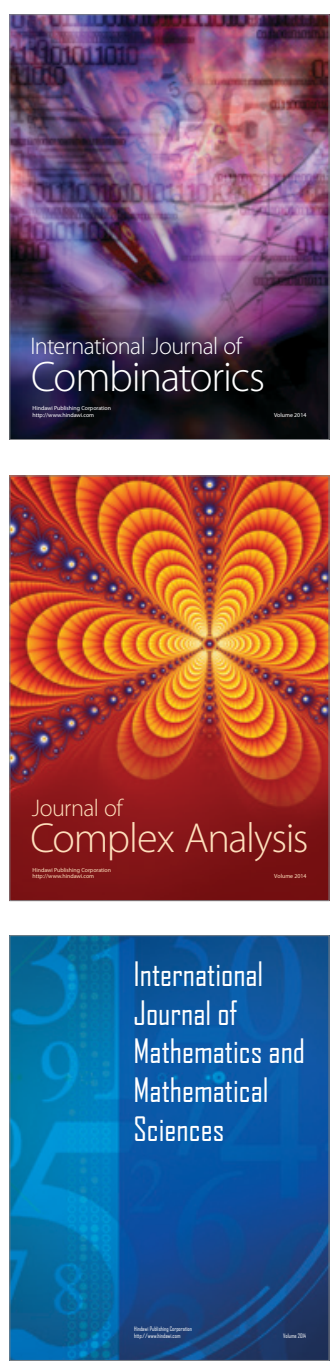
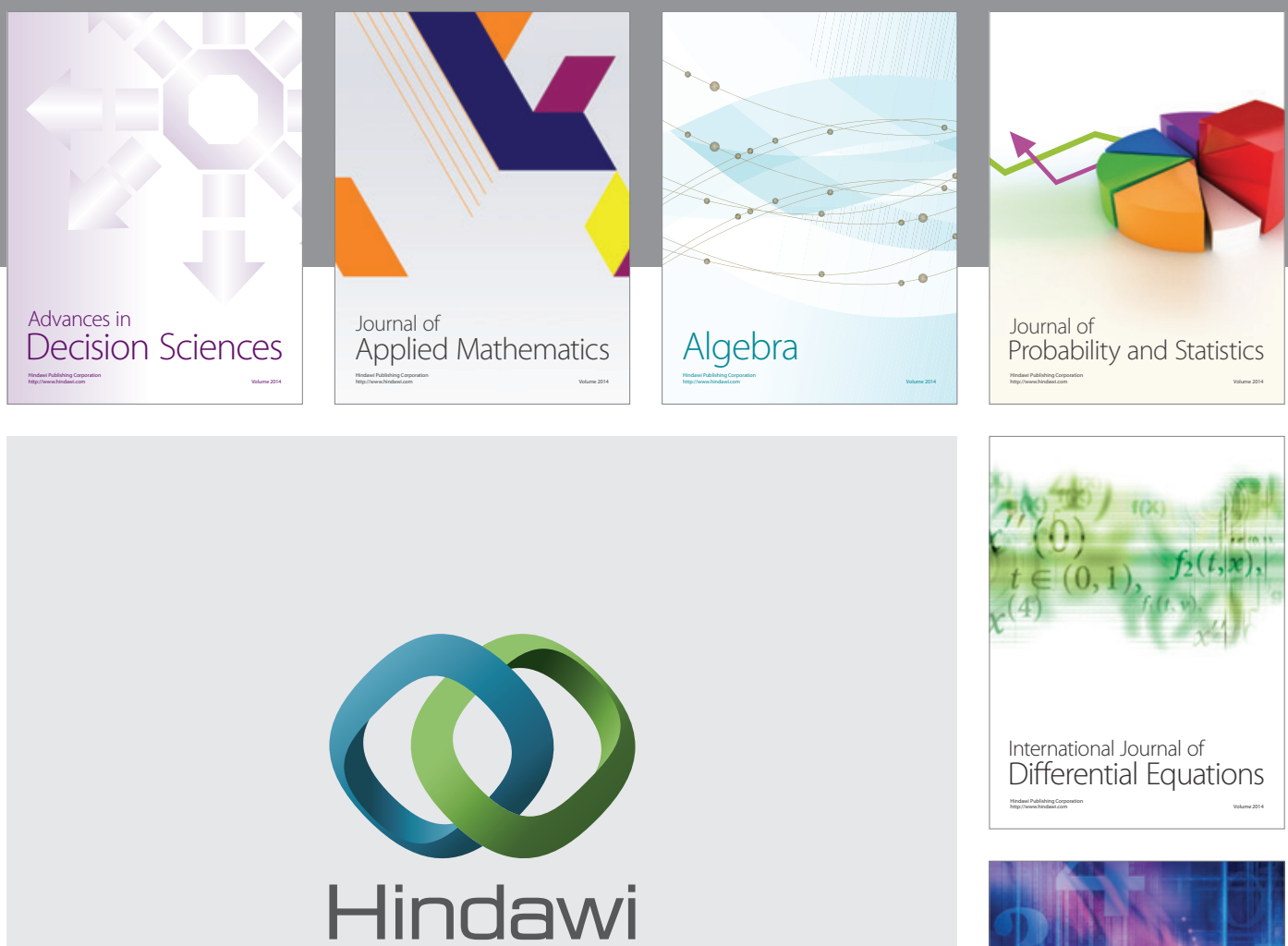

Submit your manuscripts at http://www.hindawi.com
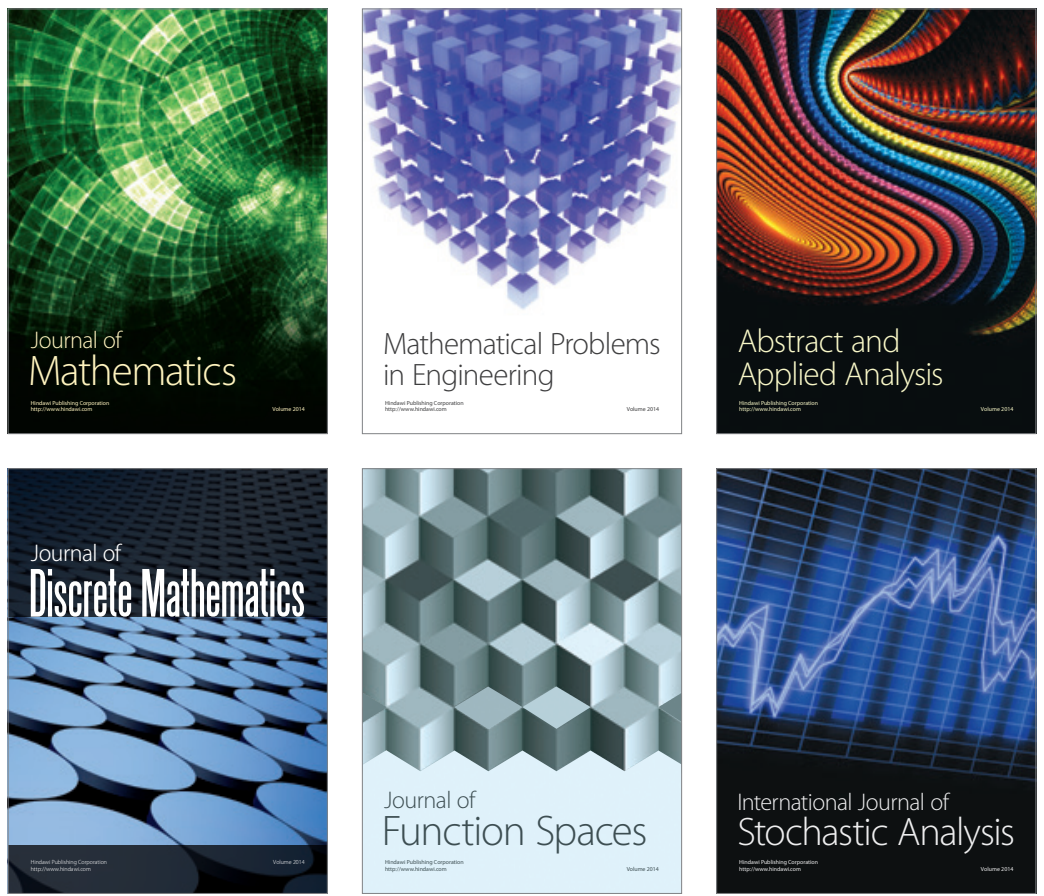

Journal of

Function Spaces

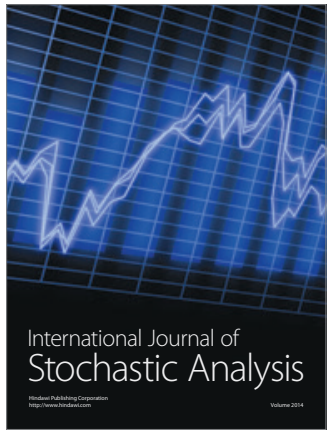

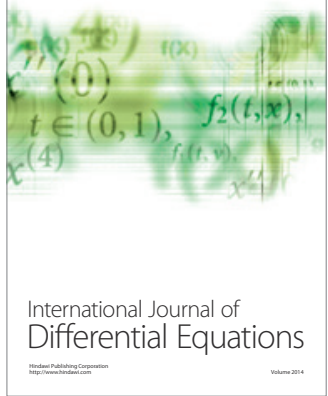
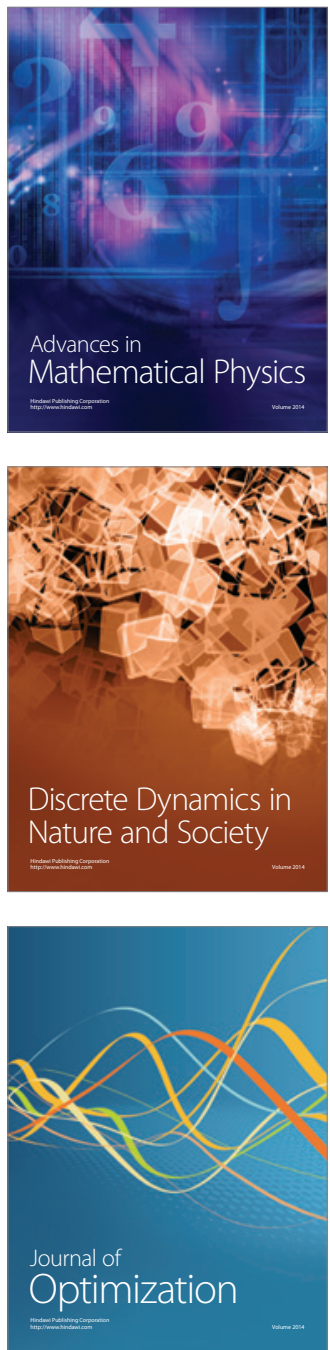